\title{
A preliminary comparison between intramedullary interlocking nail and minimally invasive plate osteosynthesis in extra-articular distal tibia fractures: a retrospective study
}

\author{
Baral $\mathrm{R}^{1}$, Raj Kandel $\mathrm{P}^{2}$ \\ 1,2 MBBS, MS(Orthopaedics and Trauma Surgery); Assistant Professor, Orthopaedic Department, Universal College of Medical Sciences, Bhairahawa, Nepal
}

Corresponding author: Dr Rajiv Baral, Assistant Professor, Orthopaedic Department, Universal College of Medical Sciences, Bhairahawa, Nepal; Tel: 977-9846036756; Email: rajibaral66@gmail.com

\begin{abstract}
Aims: To compare functional outcome between intramedullary interlocking nail (IMIL) versus minimally invasive plate osteosynthesis (MIPO) in the distal one-third extra-articular tibia fracture.

Patients and methods: We retrospectively analysed 42 cases (21 each) of the IMIL nail versus locking plate by MIPO technique. All patientswere followed for at least one year since the last case operated. This study included closed distal tibia fractures above $4 \mathrm{~cm}$ to $10 \mathrm{~cm}$ and fibula fractures. Fibula fractures within $7 \mathrm{~cm}$ of the tibial plafond were fixed with Kirschner wire (K-wire). We excluded all comminuted open fractures, intra-articular extension, and isolated fracture of the tibia from the study. Foot function index, union time, rotational and angular malalignment, rate of infection, secondary interventions and complications were compared between the groups.

Results: All patients were retrospectively analysed. Coronal $(P=0.259)$ and sagittal plane malalignment $(P=0.147)$, distraction $(P=0.147)$, rotation ( $P=0.147)$, delayed union ( $P=0.549)$, non-union ( $P=0.311)$, infection $(P=0.549)$, malunion $(P=0.147)$, amputation ( $P=0.311)$, secondary intervention $(P=0.116)$, and foot function index $(P=0.217)$ were all similar between the groups. Time to union was earlier in the MIPO group ( $\mathrm{P}=0.033)$. We, however, used the MIPO technique in the younger age groups $(P=0.042)$. Coronal plane deformity in both groups was associated with a higher rate of complications, which was also statistically significant $(P=0.012)$.
\end{abstract}

Conclusion: Our study suggests that similar outcomes appear through IMIL nail and MIPO of distal extra-articular tibia fractures. Time to union was faster in the MIPO cases, but other factors, such as age, might have played a role.

Level of evidence: Level 3

Key words: intramedullary interlocking nail, minimally invasive plate osteosynthesis, alignment, distal tibia

Citation: Baral R, Raj Kandel P. A preliminary comparison between intramedullary interlocking nail and minimally invasive plate osteosynthesis in extra-articular distal tibia fractures: a retrospective study. SA Orthop J 2017;16(4):54-58. http://dx.doi.org/10.17159/2309-8309/2017/v16n4a9

Editor: Prof Anton Schepers, University of the Witwatersrand

Received: September 2016 Accepted: March 2017 Published: November 2017

Copyright: @ 2017 Baral R, Raj Kandel P. This is an open-access article distributed under the terms of the Creative Commons Attribution Licence, which permits unrestricted use, distribution and reproduction in any medium, provided the original author and source are credited.

Funding: None declared.

Conflict of interest: The authors declare that there is no conflict of interest regarding the publication of this paper. 


\section{Introduction}

Distal tibia fractures are one of the commonest fractures that present in the emergency department. There is always a dilemma about treatment. Surgeons treat these fractures with a closed intramedullary interlocking nail (IMIL), open reduction and internal fixation with dynamic compression plate, external fixation, and minimally invasive plate osteosynthesis (MIPO). ${ }^{1,2}$ The aim of treating a fracture is to produce a stable construct which allows early mobilisation and weight-bearing, but with minimal complications. ${ }^{3}$ Post-operative axial rotational malalignment and distraction is usually more common in the IMIL group, leaving patients dissatisfied even when the degree of displacement is within the acceptable range. The overall outcome of both treatment groups is the same. We undertook this study to compare these two treatment options in our setting.

\section{Patients and methods}

This study wasconducted at the Universal College of Medical Sciences, Bhairahawa, Nepal. We consecutively analysed 42 cases (21 each) of intramedullary interlocking nail (IMIL) versus the locking plate by a minimally invasive plate osteosynthesis (MIPO) technique. This study period extended from 15 January 2013 to 16 January 2015 , with the last case being followed up for one year. The ethics committee of the hospital gave approval of the study and informed consent was taken from each patient. We included closed distal tibial fractures $4 \mathrm{~cm}$ to $10 \mathrm{~cm}$ above the ankle with fibula fractures within $7 \mathrm{~cm}$ of the tibial plafond. We used K-wires in the fibula fractures, which act as an intramedullary device. All comminuted open fractures, intra-articular extension, and isolated fracture of the tibia were excluded from the study. The sample size was calculated by standardised effect size, which was 0.9 , with $\alpha=0.005$ and $B=0.20$.

Fractures were classified according to the $A O$ classification. We included $42 \mathrm{~A}_{1}$ and $43 \mathrm{~A}_{3}$ fractures in this study. The minimally invasive fixation technique was used for plate osteosynthesis under fluoroscopy and tourniquet. A longitudinal incision of $5 \mathrm{~cm}$ was made over the medial malleolus with a meticulous dissection including the preservation of the saphenous vein and nerve, and a tunnel was made through which the plate glided smoothly towards the proximal fragment. Another $2.5 \mathrm{~cm}$ incision was made on the skin at the proximal part of the plate. The rest of the procedure was completed with a stab incision under fluoroscopy with longitudinal traction and below the ankle joint. If the reduction was difficult, we would apply pointed reduction forceps. We performed nailing by closed technique on a fracture table with the patient in the supine position, with the affected leg hanging down from the table. The leg was returned to the surface of the table in a flexed position after the insertion of the nail. The distal segment was controlled and distal locking performed by the anteroposterior fluoroscopic guided method.

Union was defined as the formation of callus on at least three cortices and clinically by the lack of pain on weight-bearing without assistance. Malunion was defined as varus or valgus greater than $5^{\circ}$ in the coronal plane (anteroposterior X-ray), or recurvatum or procurvatum greater than $10^{\circ}$ in the sagittal plane (lateral $X$-ray) or external or internal rotation greater than $10^{\circ}$ (physical examination). ${ }^{4}$ We recorded any complication during the surgery and follow-up. Delayed wound healing and superficial infection were defined as persistent drainage from the wound for at least two days, or separation of wound edges to a width $>1 \mathrm{~cm}$ and a length $>1 \mathrm{~cm} .{ }^{5}$ Moreover, the rotation was assessed by comparing the thigh-foot angle between the two legs.

Statistical analysis was performed by using a statistical package for Social Sciences (SPSS-20). Continuous variables were stated as the mean, median and standard deviation, and categorical variables as percentages and frequency distribution. An independent sample t-test after normality testing compared continuous variables between independent groups. The chi-square test was used to compare the categorical data. A value of $p<0.05$ was considered statistically significant.

\section{Results}

We included 42 cases within a minimum follow-up of one year, during which period all cases were united. The mean age of patients in the IMIL and locking plate groups was 37.38 and 46.116 years, respectively, with a standard deviation of \pm 12.188 and \pm 16 . 116 respectively. There were 12 males and nine females in the IMIL group, and 13 males and eight females in the locking plate group. We included 20 transverse, 16 spiral, and six oblique fractures.

Coronal plane malalignment was found in six cases in the IMIL group whereas three locking plates had this type of malalignment. Coronal plane malalignment in the IMIL group is statistically similar to the MIPO group $(p=0.259)$. Only two cases operated with locking plates had sagittal plane malalignment but we could not find this type of malalignment in the nailing group. This was not statistically significant $(p=0.147)$. There were two cases of distraction in the IMIL group after fixation, which was not statistically significant ( $P=0.147)$. Malrotation was found in two cases of IMIL which was functionally insignificant after the complete union ( $P=0.147)$.

All cases united with or without secondary intervention in the IMIL and locking plate groups with a mean of 17.95 weeks \pm 6.168 and 15.95 weeks \pm 3.879 respectively (range12-40 weeks). MIPO cases united earlier than IMIL cases, which was statistically significant $(\mathrm{p}=0.033)$ (Figures 1 and 2). A total of 34 fractures were associated with the lower third fibula fracture. K-wires were fixed in 23 fibula fractures by closed, minimally invasive as well as an open technique (Table I).

The coronal plane deformity was related to a higher complication rate, which was statistically significant $(p=0.012)$ (Table II). A total of seven cases of IMIL had complications. Out of these, there were two cases of delayed union, two cases of malunion, and one case each of non-union, infection and amputation. Patients with locking plates had three complications. These were one delayed union and two superficial infections. The rate of infection was similar between the groups ( $\mathrm{P}=0.549)$ (Table III).

In the second month, three patients complained of foot pain, which disappeared after removal of the fibular K-wire. One patient underwent amputation after IMIL because of vascular insufficiency. The patient had taken steroids for a long time and had a Cushingoid appearance. The pre-operative skin was thin, shiny and pigmented whereas the post-operative skin had turned black. Later Doppler ultrasonography showed venous thrombosis. Two patients in the locking plate group had insertion-site infection with wound gaping at three months after fixation which subsided after implant removal. The locking plate was removed after achieving complete union. An infection occurred in the fourth month around the distal screw in one patient of the IMIL group, with purulent discharge; when treated with antibiotics and dressings, it also subsided after implant removal, with a complete union at the fracture site. In two patients with locking plate fixation, there was difficulty in implant removal due to screw slippage, so half of the plate was left within the bone. Anterior knee pain was found in five cases of IMIL ( $P=0.017)$. Those patients with anterior knee pain did not complain of pain after removal of the implants.

Out of these 42 cases, six of IMIL and two of locking plate fixation needed secondary intervention. All six IMIL cases needed dynamisation, and union was achieved at the expected time in five cases. One case of IMIL went into non-union even after dynamisation, which was later supplemented with bone graft and locking plate. 
Table I: Patient variables in the IMIL and MIPO groups

\begin{tabular}{|c|c|c|c|c|}
\hline \multirow{2}{*}{\multicolumn{2}{|c|}{ Variables }} & \multicolumn{2}{|c|}{ Treatment } & \multirow{2}{*}{$P$ value } \\
\hline & & IMIL & MIPO & \\
\hline \multicolumn{2}{|l|}{ Age } & $37.38 \pm 12.188$ & $46.116 \pm 16.116$ & 0.042 \\
\hline \multirow{2}{*}{ Sex } & Male & 12 & 13 & 0.753 \\
\hline & Female & 9 & 8 & \\
\hline \multirow{4}{*}{ Malalignment (yes/no) } & Coronal & $6 / 15$ & $3 / 18$ & 0.259 \\
\hline & Sagittal & $0 / 21$ & 2/19 & 0.147 \\
\hline & Rotation & $2 / 19$ & $0 / 21$ & 0.147 \\
\hline & Distraction & $2 / 19$ & $0 / 21$ & 0.147 \\
\hline \multicolumn{2}{|l|}{ Union time } & $17.95 \pm 6.168$ & $15.95 \pm 3.879$ & 0.033 \\
\hline \multirow{6}{*}{ Complication (yes/no) } & Delayed union & 2/19 & $1 / 20$ & 0.549 \\
\hline & Non-union & $1 / 20$ & $0 / 21$ & 0.311 \\
\hline & Infection & $1 / 20$ & $2 / 19$ & 0.549 \\
\hline & Malunion & $2 / 19$ & $0 / 21$ & 0.147 \\
\hline & Amputation & $1 / 20$ & $0 / 21$ & 0.311 \\
\hline & Anterior knee pain & $5 / 16$ & $0 / 21$ & 0.017 \\
\hline \multicolumn{2}{|c|}{ Secondary intervention (yes/no) } & $6 / 15$ & $2 / 19$ & 0.116 \\
\hline \multicolumn{2}{|l|}{ Foot function index } & $24.90 \pm 12.401$ & $20.76 \pm 8.654$ & 0.217 \\
\hline
\end{tabular}

IMIL: Intramedullary interlocking nail

MIPO: Minimal invasive plate osteosynthesis

AOFAS: American Orthopaedic Foot and Ankle Society Score

Table II: Comparing coronal plane deformity associated with complications

\begin{tabular}{|c|c|c|c|c|}
\multirow{2}{*}{} & \multicolumn{2}{|c|}{ Complications } & \multirow{2}{*}{ P } \\
\cline { 2 - 5 } & & Yes & No & \\
\hline \multirow{2}{*}{ Coronal } & Yes & 5 & 4 & 0.012 \\
\hline & No & 5 & 28 & \\
\hline
\end{tabular}

Table III: Complications in the IMIL and MIPO groups

\begin{tabular}{|l|c|c|c|c|}
\hline \multirow{2}{*}{} & \multicolumn{2}{|c|}{ Group } & \multirow{2}{*}{ P } \\
\cline { 2 - 5 } & IMIL & MIPO & \\
\hline \multirow{2}{*}{ Coronal } & Yes & $7(16 \%)$ & $3(7.1 \%)$ & 0.147 \\
\hline Total & No & 14 & 18 & \\
\hline
\end{tabular}

There were two cases of locking plate with infection over the wound which subsided after removal of the implant and antibiotic coverage. A secondary intervention was not statistically significant between the two groups $(p=0.116)$. Foot function index determined functional outcome. ${ }^{6}$ The mean foot function index in the IMIL group was $24.90 \pm 12$, while in the MIPO group it was $20.76 \pm 8.654$ at last follow-up. The overall foot function index was not statistically significant $(p=0.217)$.

\section{Discussion}

There is always some difficulty in choosing an implant for the treatment of distal third tibia fractures. There are certain advantages and disadvantages of each treatment (IMIL and MIPO) method based on operative time, incision length, fluoroscopy time, bleeding, difficulty in distal locking, indirect reduction, etc. ${ }^{4,5}$
Acceptable and near anatomic reduction is the goal of treatment in distal tibial fracture management. Malalignment could lead to secondary osteoarthritis of the ankle and knee joints. It is quite difficult to reduce the fracture indirectly in both treatment methods, even though more cases of malalignment were noticed in the nailing group. It is possible to achieve anatomical alignment in open reduction and internal fixation, but it has some disadvantages that interfere with fracture healing., ${ }^{5,7}, \mathrm{In}$ a prospective study by Im and Tae, nailing showed an advantage in operating time, movement and wound problems, but anatomical plates produced better alignment. ${ }^{9}$ Malrotation was better restored in the MIPO group. ${ }^{4}$ We found a slightly larger number of cases of malalignment in the IMIL group as compared to the locking plate group. The distal tibia has a circular cross-sectional area with a thinner cortex as compared to the triangular diaphysis with a thicker cortex. Moreover, the intramedullary nail designed for a tight interference fit at the diaphysis cannot provide the same stability at a distal fracture..$^{10-12}$ This malalignment is coronal as well as sagittal. Coronal plane malalignment will have more complications, which is also statistically significant $(P=0.012)$. There were two sagittally malaligned fractures treated by locking plate. Distraction and rotational malalignment were not uncommon in the IMIL group in our study. Dynamisation was carried out in those cases where inadequate callus was noted even after partial weight bearing which started six weeks after fixation. All these cases later had a good functional outcome.

Time to union directly reflects the surgical technique. Both procedures are minimally invasive, which means that fracture haematoma is maintained. Yang et al. found that the time to union was shorter in the IMIL group, but with an increase in post-operative valgus by a mean of $3.7^{\circ}$, whereas we found a shorter union time in the MIPO group ( $\mathrm{P}=0.033) .{ }^{13}$ Guo et al. reported no patients with non-union in their series and all the IMIL cases were reamed. ${ }^{5} \mathrm{Li}$ et al. reported only two patients (one patient in IMIL and another patient in the MIPO group) with non-union. ${ }^{5,8}$ We did find a difference in union time, which is unlike the studies conducted by Polat et al. and Xue et al., ${ }^{414}$ Delayed union is found in both cases, but non-union observed in only one case in IMIL group, which had coronal plane malalignment. 


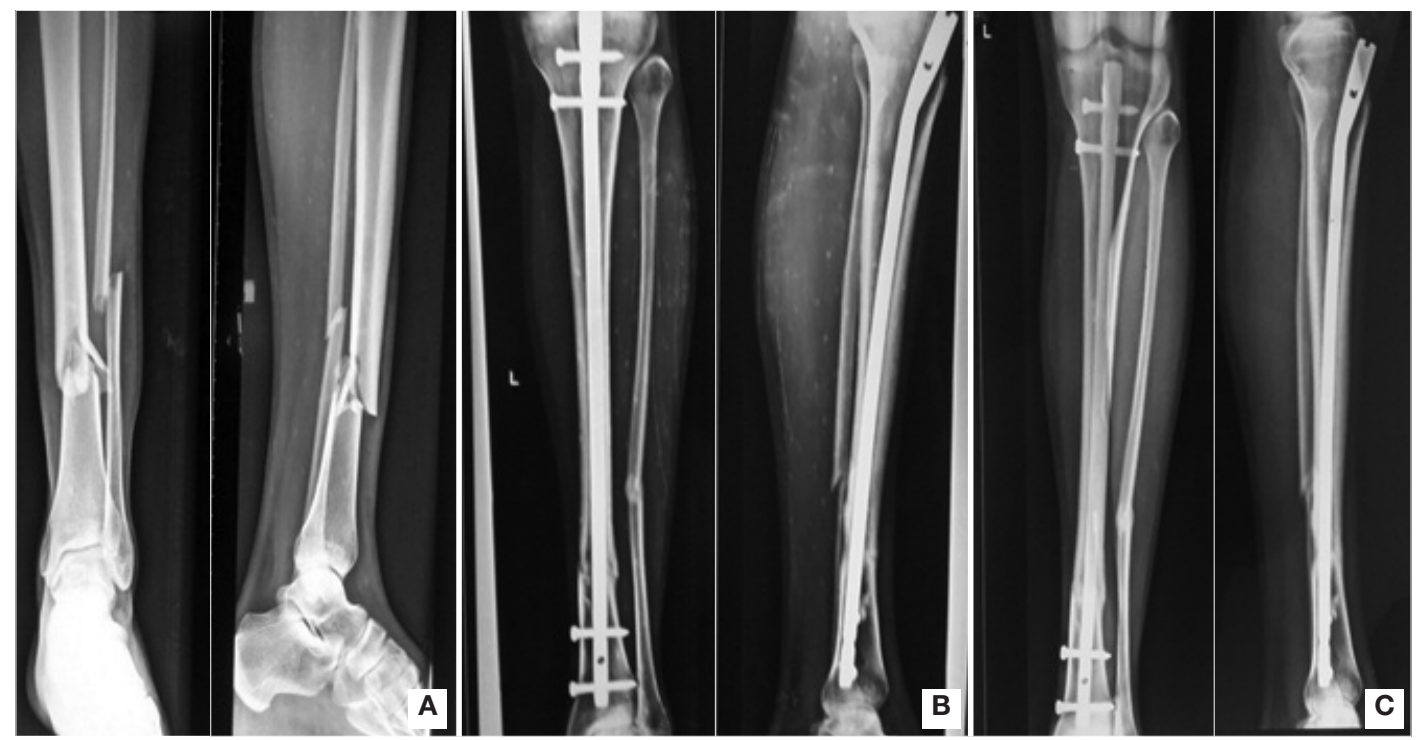

Figure 1. A patient treated with IMIL; (A) pre-operative, (B) early post-operative, and (C) final radiograph

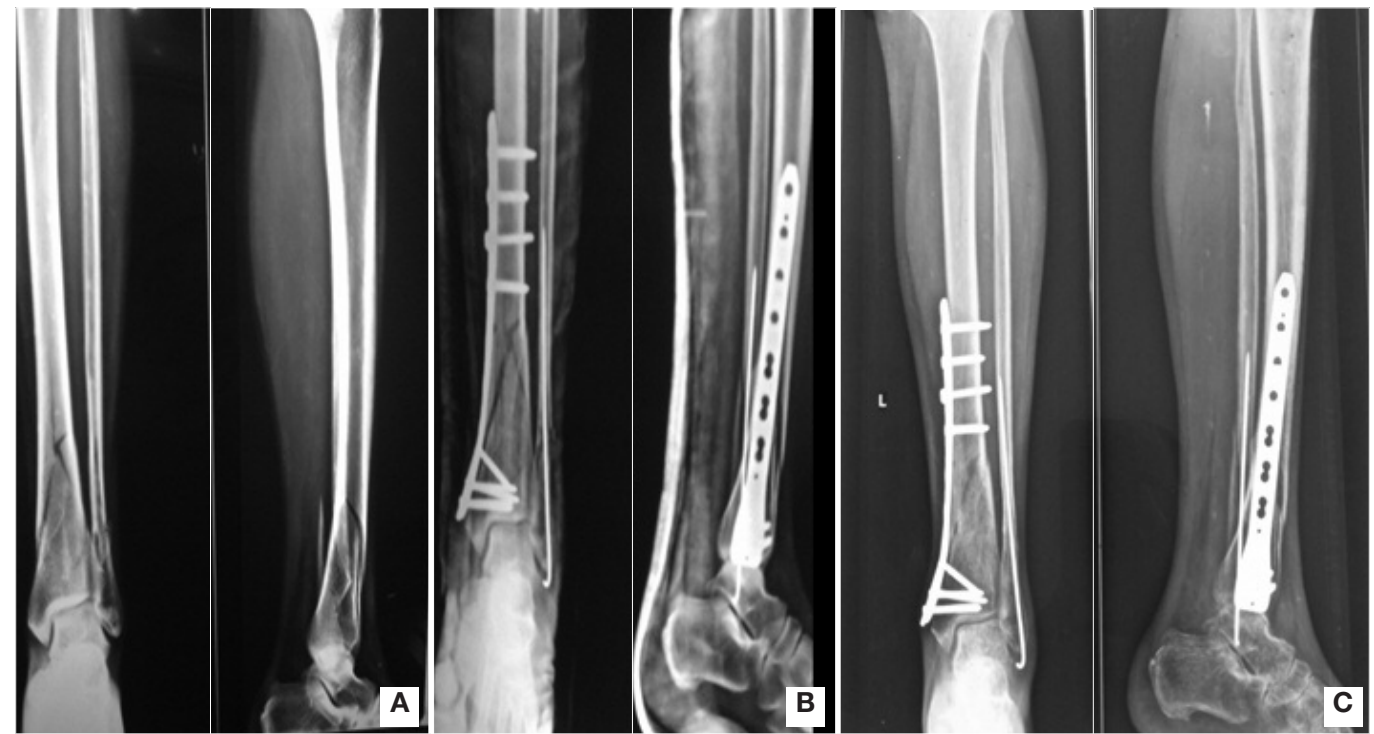

Figure 2. A patient treated with MIPO; (A) pre-operative, (B) early post-operative, (C) final radiograph

Fibular fractures within $7 \mathrm{~cm}$ of the ankle plafond are usually fixed with a plate and screws. Stable K-wire fixation is preferable according to the principle of the minimally invasive osteosynthesis technique. Concurrent open reduction and internal fixation of a fibular fracture may contribute to non-union of the tibial fracture because of a relatively larger reduction in strain across it. ${ }^{7,15} \mathrm{We}$ used K-wires as an intramedullary device to fix the fibula fractures, which were inserted closed under fluoroscopy. We used $2 \mathrm{~mm}$ $\mathrm{K}$-wires before fixation of the tibia. Two of the patients complained of pain on the lateral side on weight-bearing at one-and-a-half months, which was relieved after removal of the K-wire from the fibula. Closed K-wire insertion was difficult in two cases in each group. Consequently, we performed a minimally invasive technique using artery forceps. We did an open reduction and internal fixation in one case due to difficulty experienced in the minimally invasive technique. We did not note any case of malunion, delayed union, ankle instability or non-union in fibula fractures fixed with K-wires.

There is controversy about the need for routine removal of the implant. Guo et al. removed the plate due to skin impingement but we did not find such a problem in our study. They had some difficulty in removing the locking screws because of the stripping of the hexagonal recess and the threads of the head of the locking screw in nine patients. ${ }^{5}$ We did face difficulty during implant removal in the cases fixed with the locking plate. We used a local Indian implant (Madura ortho surge) due to cost factors. During operation, the screw slippage rate was very high. If slippage occurs in the locking plate, it is very difficult to remove. We have even left the distal portion of a plate after cutting off the rest of the implant. It was also difficult to obtain the sophisticated screw-removal instrument. We did not have any difficulty in implant removal in any case of intramedullary interlocking nail except proximal locking screw slippage, in which case it was removed by grasping with a forceps and screwdriver.

Many cases of fracture fixed by implants are not devoid of complication. The subcutaneous location and less muscle mass around the distal tibia have a poor vascular supply. The total complication rate was significantly higher for intramedullary nailing compared with plate fixation (44.5 vs $25.8 \%, \mathrm{P}<0.001) .{ }^{16}$ Although statistically not significant, the rate of complication in IMIL is higher as compared to the MIPO group (16\% vs $7.1 \%, P=0.147$ ) in our study. The rate of infection by different researchers appears similar between the MIPO and IMIL groups. ${ }^{17,18}$ Infection is found in both groups. 
Polat et al. noted one infection in the MIPO group which abated upon removal of the implants. ${ }^{4}$ Our study showed that infections decreased after the removal of the plate and nail. A patient who was on steroids for a long time with pre-operative pigmented shiny skin required an amputation after IMIL. The cause of the amputation was gangrene due to venous thrombosis identified by colour Doppler. Yang et al. compared the results of IMIL versus open reduction and plating in distal tibial fractures and reported anterior knee pain in almost half of their patients (6/13), whereas there were no patients with knee symptoms in the plating group. Two of their patients' symptoms continued after the removal of the IMIL nail. ${ }^{13}$ Other studies also list knee pain as a disadvantage of IMIL. ${ }^{4}$ Our studies showed five such cases of anterior knee pain causing functional impairment in those treated with IMIL.

People in our setup are reluctant to have a secondary intervention and follow-up is irregular. Guo et al. confirmed that reamed nailing was safe, even for distal metaphyseal fractures of the tibia, and was linked with a low risk of delayed union and no risk of non-union. ${ }^{5}$ Although the aetiology of non-union is multifactorial, it is well known that surgical technique is one of the most important determinants of union. ${ }^{4}$ There were no cases of non-union, suggesting the closed application of IMIL and MIPO for preservation of the soft tissues and fixation of the fractures was advantageous. ${ }^{19}$ We did dynamisation in some cases in the IMIL group due to delayed union and to accelerate union. One patient suffered from non-union even after dynamisation; consequently, we did a bone graft and plating. Two cases of locking plate with infection were treated with implant removal and antibiotic coverage.

Polat et al.'s study showed that both MIPO and IMIL are equally effective in terms of functional outcomes. ${ }^{4}$ Guo et al. compared MIPO and IMIL in a series of 85 patients with distal tibial fractures and reported statistically similar American Orthopedic Foot and Ankle Society Score scores in both groups. ${ }^{19}$ We could also not find any statistically significant foot function index between the two groups ( $p=0.217)$.

Our main limitation of the study was an irregular follow-up of the patient due to an unstable situation in the country. Nevertheless, we did not lose a single case to follow-up. We had to delay the operation until subsidence of swelling of the limb in the locking plate group, but, that may not have had an effect on the final outcome. We have also not compared fluoroscopy time, blood loss, incision length, and operative time between the groups. An intramedullary interlocking nail in the distal tibia is a highly demanding procedure as compared to plate osteosynthesis. It is difficult to control a distal segment fracture during IMIL insertion. If any malalignment occurs, complications are unavoidable. Thus, during operation, proper reduction during insertion, manipulation before and after distal locking, and back hammering should be done. IMIL in distal extraarticular tibial fractures has a steep learning curve to obtain a good result. If carefully performed, both techniques result in no functional consequences.

\section{Compliance with ethics guidelines}

Approval for this study was granted by the Institutional Review Committee of the Universal College of Medical Sciences, Bhairahawa, Nepal.

\section{References}

1. Nork SE, Schwartz AK, Agel J, et al. Intramedullary nailing of distal metaphyseal tibial fractures. J Bone Joint Surg [Am] 2005;87-A: 1213-21.

2. Borg T, Larsson S, Lindsjö U. Percutaneous plating of distal tibial fractures: preliminary results in 21 patients. Injury 2004;35:608-14.

3. Little NJ, Verma V, Fernando C, Eliott DS, Khalell A. A prospective trial comparing the Holland nail with the dynamic hip screw in the treatment of intertrochanteric fractures of the hip. J Bone Joint Surg [Br] 2008; 90-B:1073-78.
4. Polat A, Kose O, Canbora K, Yanik S, Guler F. Intramedullary nailing versus minimally invasive plate osteosynthesis for distal extra-articular tibial fractures: a prospective randomized clinical trial. J Orthop Sci. 2015 Jul;20(4):695-701.

5. Guo JJ, Tang N, Yang HL, Tang TS. A prospective randomised trial comparing closed intramedullary nailing with percutaneous plating in the treatment of distal metaphyseal fractures of the tibia. $J$ Bone Joint Surg Br. 2010;92(7):984-88.

6. Budiman-Mak E, Conrad KJ, Roach KE. The foot function index: a measure of foot pain and disability. J Clin Epidemiol. 1991;44(6): 561-70.

7. Vallier HA, Cureton BA, Patterson BM. Randomized, prospective comparison of plate versus intramedullary nail fixation for distal tibia shaft fractures. J Orthop Trauma. 2011;25(12):736-41.

8. Li Y, Jiang X, Guo Q, Zhu L, Ye T, Chen A. Treatment of distal tibial shaft fractures by three different surgical methods: a randomized, prospective study. Int Orthop. 2014;38(6):1261-67.

9. Im GI, Tae SK. Distal metaphyseal fractures of tibia: a prospective randomized trial of closed reduction and intramedullary nail versus open reduction and plate and screws fixation. J Trauma 2005;59:1219-23.

10. Kneifel T, Buckley R. A comparison of one versus two distal locking screws in tibial fractures treated with unreamed tibial nails: a prospective randomized clinical trial. Injury 1996, 27:271-73.

11. Trafton PG. Tibial Shaft Fractures. In: Skeletal trauma. 4th edn. Edited by Browner DB, Jupiter JB, Levine AN, Trafton PG, Krettek C. Philadelphia: Suanders Elsevier; 2009:2319-52.

12. Shrestha D, Acharya BM, Shrestha PM. Minimally invasive plate osteosynthesis with locking compression plate for distal dia metaphyseal tibia fracture. Kathmandu Univ Med J 2011;34(2):62-68.

13. Yang SW, Tzeng HM, Chou YJ, Teng HP, Liu HH, Wong CY. Treatment of distal tibial metaphyseal fractures: plating versus shortened intramedullary nailing. Injury. 2006;37(6):531-35.

14. Xue XH, Yan SG, Cai XZ, Shi MM, Lin T. Intramedullary nailing versus plating for extra-articular distal tibial metaphyseal fracture: a systematic review and meta-analysis. Injury. 2014;45(4):667-76.

15. Teitz CC, Carter DR, Frankel VH. Problems associated with tibial fractures with intact fibulae. J Bone Joint Surg [Am] 1980;62-A:770-76.

16. Li B, Yang $Y$, Jiang LS. Plate fixation versus intramedullary nailing for displaced extra-articular distal tibia fractures: a system review. Eur J Orthop Surg Traumatol. 2015 Jan;25(1):53-63.

17. Kwok CS, Crossman PT, Loizou CL. Plate versus nail for distal tibial fractures: a systematic review and meta-analysis. J Orthop Trauma. 2014;28(9):542-48.

18. Newman SD, Mauffrey CP, Krikler S. Distal metadiaphyseal tibial fractures. Injury. 2011;42(10):975-84.

19. Guo JJ, Yang H, Qian $H$, et al. The effects of different nutritional measurements on delayed wound healing after hip fracture in the elderly. J Surg Res 2010;159:503-8. 